DIVISION OF THE HUMANITIES AND SOCIAL SCIENCES

CALIFORNIA INSTITUTE OF TECHNOLOGY

PASADENA, CALIFORNIA 91125

Design of Tradable Permit Programs under Imprecise Measurement

John O. Ledyard

California Institute of Technology

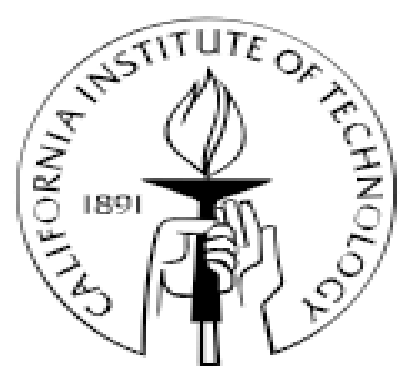

SOCIAL SCIENCE WORKING PAPER 1438

March 2018 


\title{
Design of Tradable Permit Programs under Imprecise Measurement
}

\author{
John O. Ledyard ${ }^{*}$
}

\begin{abstract}
If the measurement of production in a commons is accurate and precise, it is possible to design a tradable permit program such that, under a fairly general set of conditions, the market equilibrium is efficient for the given aggregate permit level and everyone is better off after the permit program than before. Often, however, implementation of a tradable permit system is postponed or never undertaken because an inexpensive technology able to provide accurate and precise measurements does not exist. However, there often is an inexpensive technology which accurate but not precise. I study the possibilities for the design of a tradable permit system when the measurement technology involves an imprecise, indirect measure of production that contains statistical uncertainty. To the best of my knowledge, this has not been studied before.

As one might expect, imprecise measurement can lead to inefficiency and prevent voluntary participation. But there are positive results. If measurement errors are proportional to use, it is possible to design so that aggregate output is efficiently allocated. Also, it is possible to calculate a set of individual firm lump-sum subsidies to attain voluntary participation.
\end{abstract}

Keywords: quota, permits, rights, tradable, imprecise measurement, voluntary participation, imperfect enforcement

${ }^{*}$ I thank the Max Factor Family Foundation in partnership with the Jewish Community Foundation of Los Angeles for its financial support of this project.

$\dagger$ Address: Division of the Humanities and Social Sciences, California Institute of Technology, 1200 E. California Blvd., Pasadena, CA 91125. Email:jledyard@caltech.edu 


\section{Introduction}

The formal approach to mechanism design began with Hurwicz's 1960 paper. He recognized that the information about the economic environment, such as technological possibilities, preferences, and endowments, is dispersed among economic agents and that the "informational tasks entailed by the mechanism imply costs in real resources used to operate the mechanism" (Hurwicz and Reiter 2006, p.1). He then focused on those informational tasks and searched for mechanisms that produced an efficient resource allocation and were informationally efficient in the sense that the messages were smaller that those of other possible mechanisms that produced an efficient resource allocation. In his 1972 paper, Hurwicz took mechanism design to the next level by introducing the concept of incentive compatibility. Soon after, in 1973, Gibbard introduced the Revelation Principle which led theorists to focus on direct mechanisms, mechanisms whose messages are everything an agent knows about the environment, and to ignore the "informational tasks" entailed by such a mechanism. I believe more attention needs to be put on the costs of acquiring the information necessary to attain a measure of resource efficiency and the trade off between the two types of efficiency. In this paper, I take a very small step in that direction.

I look at one of the informational tasks involved in cap and trade programs. Such programs possess a degree of informationally efficiency since they employ the price mechanism to communicate needed information about the environment. ${ }^{1}$ But there is another, usually ignored, part of the problem identifying the size of the output decisions of producers in a commons. In order to enforce the rules of a cap and trade program, it is necessary to compare that output to the permits held by the agents. But it is often the case in practice that it is difficult or impossible to achieve valid measurement of that output. Instead imprecise measurement is possible and less expensive. In this paper I pursue what is lost, if anything, by relying on that imprecise measurement when using cap and trade to manage a commons.

The tragedy of the commons is well-known: an unmanaged, common resource will be over used and the benefits from its use will be lower than would be possible under a benevolent dictator. It is also well-known that, if use can

\footnotetext{
${ }^{1}$ I will side-step adverse selection incentive problems by assuming competitive behavior on the part of the economic agents.
} 
be accurately and precisely measured, it is possible to design a tradable permit program such that, under a fairly general set of conditions, the permit market equilibrium allocation is efficient for the given aggregate permit level and everyone is better off after the permit program than before. I will refer to such a program as satisfactory.

In practice, however, the implementation of a tradable permit program is often postponed or never undertaken because there does not exist an inexpensive technology able to measure violations accurately and precisely. However, sometimes there is an inexpensive technology available that can measure violations imprecisely. That is, there is random measurement error. Examples of imprecise measurement in commons problems are easy to find. I look at only two: fisheries and ground water.

In the management of fisheries, it is the catch is that is often permitted. The easiest and most direct way to measure the catch is at the landing where the catch is unloaded and can easily be weighed. But that is actually only an inaccurate and imprecise measure of what has really been caught. For example, high grading, keeping the best and tossing the rest, would mean more fish are caught than measured. The best place to measure the actual catch is at the point of catch, but that is remote. Nevertheless, a variety of methods have been employed to try to get such measurements. Remote sensing through satellites, onboard human observers and/or surveillance cameras are some of the technologies used. While these seem to improve measurement, there are still errors. The measurement is imprecise.

In the management of ground water, it is important to know the amount of water pumped from an aquifer. The obvious way to measure it is to place a meter at the pump. But in many situations, meters do not exist. Meters are costly and the manpower to read them is expensive. There have been several alternative measurement schemes proposed to be used in place of direct metering. One is to measure the electricity used by the pumps. The Turlock Irrigation District in California uses this method. ${ }^{2}$ Another method is to use remote sensing by satelite or unmanned drones, using evapotranspiration as

\footnotetext{
${ }^{2}$ See also Zekri (2009) for a deeper discussion of this method.
} 
an indirect measure of water use. ${ }^{3}$ Of course, both these technologies have errors in measurement. The measurement is imprecise.

To the best of my knowledge, the implications of imprecise measurement for permit markets have not been studied before. In the literature, measurement is always perfect - precise and accurate. Some studies have considered the case when, although the measurement is perfect, it is only done with some probability - thereby lowering the costs. This is often called random or imperfect monitoring. ${ }^{4}$ The main conclusion of this research is that, with risk neutral producers, if the penalty rate per unit violation is high enough then the equilibrium allocations in a competitive permit market will be exactly the same as under perfect measurement. As long as the expected cost of a violation is high enough, producers in a commons will chose to hold permits exactly equal to their planned output. Because of that, the combination of perfect measurement and random monitoring can lead to a satisfactory program.

In this paper, I study whether imprecise measurement could be the basis for a satisfactory permit program. Unfortunately and perhaps not surprisingly, the answer is no. It is useful to understand why. With imprecise measurement, producers will buy more permits than their actual output in order to insure against the potential that measurement will indicate higher production than is actually the case. This leads to two types of resource inefficiency. One occurs because aggregate output will be less than the aggregate amount of permits. The second occurs if the errors in measurement are biased. If errors are not proportional to production, then aggregate output will not be efficiently allocated. Imprecise measurement can also make it difficult or impossible to guarantee that all producers will be at least as well off as they were before the program was put into place. This because the aggregate cost of holding permits to insure against mis-measurement can be greater than the increase in aggregate benefits from better management of the commons. This can cause political difficulties.

\footnotetext{
${ }^{3}$ See Water Education Foundation (2015) for a discussion of this method.

${ }^{4}$ See Malik (1990), Malik (1992), Stanlund and Dhanda (1999), Stanlund (2007), Murphy and Stanlund (2007), and Stanlund et. al. (2008).
} 
In spite of these negative findings, there are some positive results. First, if measurement errors are proportional to use, then it is possible to design a tradable permit program with imprecise measurement such that the equilibrium aggregate output is efficiently allocated. Second, there are easily calculated subsidies that allow the design of a tradable permit program that guarantees that producers will be better off than they were before the program. Third, as the precision of the measurement approaches perfect accuracy, the equilibrium of the market with imprecise measurement approaches what it would be with perfect measurement and the size of the subsidies necessary to guarantee a Pareto-improvement decline to zero.

\section{The Commons}

A collection of producers, named $i=1, \ldots, N$, are involved in a commons. Each chooses a level of production, $q_{i} \cdot{ }^{5}$ A producer's economic profits are $b_{i}\left(q_{i}, Q\right)$ where $Q=\sum_{i} q_{i}$. The fact that aggregate output affects an individual's profits creates the externality that is at the heart of the commons problem. There is a set of standard assumptions that guarantees this model of the commons is well-behaved.

Assumption 1 (Regular Commons).

(i) $b_{i}\left(q_{i}, Q\right)$ is strictly concave and increasing in $q_{i}$, decreasing in $Q$, and continuously differentiable in $q_{i}$ and $Q$.

(ii) $\lim _{x \rightarrow 0} b_{i q}(x, Q)=\infty .^{6}$

(iii) $b_{i q Q} \leq 0, \forall i$.

Assumption 1(i) is standard. Assumption 1(ii) ensures that no producer will ever want to drop out. This is not necessary for many of the results in this paper but does make them a little cleaner. Assumption Assumption 1(iii) is similar to a single crossing condition. It ensures that aggregate demand is downward sloping.

\footnotetext{
${ }^{5}$ The model would be essentially the same if the producers were choosing an input.

${ }^{6} \mathrm{I}$ use the following notation for derivatives of functions: $f_{x}=\partial f(x, y, z) / \partial x$ and $f_{x y}=\partial^{2} f(x, y, z) / \partial x \partial y$. The index $\mathrm{i}$ is the name of a producer and is not a variable.
} 
I assume throughout that producers behave competitively in the commons. That is, each producer acts as if their individual choice of production level will not affect aggregate production.

In the absence of collective management, autarky reigns.

Definition 1 (Autarkic Equilbrium).

An Autarkic Equilibrium is $\left(q^{a}, Q^{a}\right)$ where (i) $q_{i}^{a}$ solves $\max _{q} b_{i}\left(q, Q^{a}\right), \forall i$ and (ii) $Q^{a}=\sum_{i} q_{i}^{a}$.

Economic efficiency is a standard benchmark and a desirable target for public policy.

Definition 2 (Efficient Allocation).

An allocation $\tilde{q}$ is Efficient if and only if $\tilde{q}$ solves $\max _{q, Q} \sum_{i} b_{i}\left(q_{i}, Q\right)$ subject to $Q=\sum_{i} q_{i}$.

It is well known that Autarkic equilibria are generally not efficient. Thus, there is the opportunity to design a policy that will guide the producers on the commons to higher aggregate profits. A tradable permit program can be one such policy.

In Section 3, I provide some basic results for a permit market with accurate measurement. This material is reasonably well known and is provided to introduce the reader to the notation, concepts and prior results that I will later refer to. Section 4 contains the main results of this paper.

\section{Tradable Permits with Valid Measurement}

A tradable permit program specifies an aggregate level of permits, L, allocates it to the producers, requires the producers to keep their production level to no 
more than the permits than they hold, and allows trading of those permits. The initial allocation of permits is $l^{o}$, where $\sum l_{i}^{o}=L$.

A valid measurement technology is both accurate and precise. With valid measurement of $q$, a tradable permit program can be enforced by monitoring and imposing a penalty for producing more than the permits held. I assume that the penalty is a function only of the level of violation $v_{i}=q_{i}-l_{i}$. This is standard in the literature. ${ }^{7}$ The penalty to be paid by $i$ is indicated by $P\left(v_{i}\right)$. One typical and simple form of the penalty function has $P\left(v_{i}\right)=\max \left\{0, a v_{i}\right\}$ where $a$ is a positive constant. I allow a wider range of possibilities.

I assume producers behave competitively in the permit market. ${ }^{8}$ Let $p$ be the market price of permits.

Definition 3 (Market Equilibrium under Valid Measurement).

A Permit Market Equilibrium under Valid Measurement is $\left(q^{*}, v^{*}, p^{*}, Q^{*}\right)$ such that,

(i) each producer $i$ chooses $\left(q_{i}^{*}, v_{i}^{*}\right)$ to solve

$$
\max _{\left(q_{i}, v_{i}\right)} b_{i}\left(q_{i}, Q^{*}\right)-p^{*}\left(q_{i}-v_{i}-l_{i}^{o}\right)-P\left(v_{i}\right)
$$

(ii) $Q^{*}=\sum_{i} q_{i}^{*}$, and

(iii) $\sum_{i} q_{i}^{*}-v_{i}^{*}=L$.

In Definition 3, $(q, Q)$ and $v$ can be solved for independently since they are separable. We use this fact to define a supply of output and a demand for violations.

\footnotetext{
${ }^{7}$ Of course, $P$ could depend on more than just $v_{i}$. For example, $P$ could depend on the percentage violation so that the penalty is $P\left(q_{i} / l_{i}\right)$. But when $\partial P / \partial q_{i}+\partial P / \partial l_{i} \neq 0$, permit market equilibria will generally not be efficient. Thus, the design choice is usually $P\left(v_{i}\right)$.

${ }^{8}$ This is rarely true in practice. Even if there is a large number of producers, most extant permit markets are disorganized and thinly traded. They tend to violate the Law of One Price and, therefore, traders' behaviors are not really competitive. There are ways to design a trading mechanism to avoid this, but that rarely happens.
} 
Definition 4 (Supply and Demand under Valid Measurement).

(a) Supply under valid measurement is $\left[q^{V}(p), Q^{V}(p)\right]$ where

$q_{i}^{V}(p) \in \arg \max _{q_{i}} b_{i}\left(q_{i}, Q^{V}(p)\right)-p q_{i}$ and $Q^{V}(p)=\sum_{i=1}^{N} q_{i}^{V}(p)$.

(b) Demand under valid measurement is $\left[v^{V}(p), V^{V}(p)\right]$ where

$v_{i}^{V}(p) \in \arg \max _{v} p v^{i}-P\left(v_{i}\right)$ and $V^{V}(p)=\sum_{i=1}^{N} v_{i}^{V}(p)$.

The market equilibrium price satisfies $Q^{V}\left(p^{*}\right)-V^{V}\left(p^{*}\right)=L$. To ensure the market is well-behaved, I impose a regularity assumption.

Assumption 2 (Regular Accurate Measurement).

(i) $P\left(v_{i}\right)=0$ on $v_{i} \leq 0 . P\left(v_{i}\right)$ is convex, increasing, and continuously differentiable on $v_{i} \geq 0$.

(ii) $L>Q^{V}\left(P_{v}\left(0^{+}\right)\right) \cdot{ }^{9}$

Assumption 2 (i) is a slight generalization of the linear penalty, $\max \left\{0, a v_{i}\right\}$.

Assumption 2 (ii) is needed to ensure that the penalty is strong enough so that producers do not want violations in equilibrium.

Result 1. Under Assumptions 1 and $2, Q_{p}(p)<0$ and $V_{p}(p) \geq 0 .{ }^{10}$

The geometry of this market is displayed in Figure 1 in Section 7.1.

\subsection{Efficiency and Political Viability}

Permit markets mimic competitive markets which, under the appropriate conditions, produce allocations that are efficient. But permit markets do not necessarily produce efficient allocations unless the supply of permits, $L$, is exactly right. Instead, permit markets produce allocations that are efficient given the total amount of permits, $L$.

\footnotetext{
${ }^{9} P_{v}\left(0^{+}\right)=\lim _{x \rightarrow 0^{+}} P_{v}(x)$.

${ }^{10}$ The proofs are omitted since they are standard and straight-forward. For the details see, e.g., Ledyard (2018).
} 
Definition 5 (Efficiency given X).

The allocation $\tilde{q}$ is Efficient given $X$ if and only if $\tilde{q}$ solves $\max _{q} \sum_{i} b_{i}\left(q_{i}, X\right)$ subject to $\sum_{i} q_{i}=X$.

If the total number of permits, $L$, is chosen appropriately, aggregate profits after the program is implemented will be higher than before. But producers won't receive those increases unless the program is actually adopted and implemented. A satisfactory program must be politically viable.

If the permit program can be designed in a way that all producers are better off after the introduction of the permit program than they are in the autarkic equilibrium, then the program will be more likely to be adopted. In the language of mechanism design, such a property of the design is called voluntary participation or individual rationality. The well known result is that all producers will be better off at the permit market equilibrium than in autarky as long as the distribution of the initial permits, $l^{\circ}$, is such that all producers are at least as well off at $l_{i}^{o}$ as they are in autarky. ${ }^{11}$

Result 2. Let $\left(q^{*}, v^{*}, p^{*}, Q^{*}\right)$ be a permit market equilibrium. Under Assumptions 1 and 2:

A) Efficiency

(i) $q^{*}$ is efficient given $L, Q^{*}=L$, and $v^{*}=0 .{ }^{12}$

(ii) The permit market equilibrium is independent of the initial distribution, $l^{\circ}$, and only depends on $L$.

\section{B) Voluntary Participation}

(i) For any initial allocation of permits, $l^{o}$, such that $b_{i}\left(l_{i}^{o}, L\right)>b_{i}\left(q^{V}, Q^{V}\right), \forall i$, it will be true that $b_{i}\left(q_{i}^{*}, Q^{*}\right)-p^{*}\left(q_{i}^{*}-v_{i}^{*}-l_{i}^{o}\right)-P\left(v_{i}^{*}\right)>b_{i}\left(q_{i}^{V}, Q^{V}\right), \forall i$.

(ii) Let $q^{e}$ be efficient given $L$ and $Q^{e}=\sum_{i} q_{i}^{e}$. There exists an $l^{o}$ satisfying (i) if and only if $\sum_{i} b_{i}\left(q_{i}^{e}, Q^{e}\right)>\sum_{i} b_{i}\left(q_{i}^{V}, Q^{V}\right)$.

\footnotetext{
${ }^{11}$ Since it is rare that such a distribution is unique, there may still be serious political bargaining over the allocation of $\mathrm{L}$.

${ }^{12}$ If the penalty is weak so that $L<Q^{V}\left(P_{v}\left(0^{+}\right)\right)$, then $v_{i}^{*}>0$ and $Q^{*}>L$. In this case, $q^{*}$ is not efficient given $L$. But $q^{*}$ will be efficient given $Q^{*}$. See, e.g., Malik (1990).
} 
The proofs are omitted since they are standard and straight-forward. ${ }^{13}$

Result (i) holds because the producer has been put into an initial position at least as good as autarky and, by choosing $q_{i}=l_{i}^{o}$, she can protect that position. Anything she then decides to do will be at least as good. If the commons effect of $Q$ is large enough, as in fisheries, then letting $l^{\circ}$ be based on autarkic output, so that $l_{i}^{o}=\frac{q_{i}^{a}}{Q^{a}} L$, will often be sufficient.

Result (ii) holds because of the quasi-linearity of profits. Locally, if $L<Q^{V}$, there exists an $l^{o}$ satisfying (i).

To summarize, with a valid measurement technology it is possible to design a tradable permit program such that, under a fairly general set of conditions, the market equilibrium is efficient for the given permit total and everyone is better off than with autarky.

\section{Tradable Permits with Imprecise Measure- ment}

As shown in the previous section, under ideal conditions, the benefits of using a tradable permit system to manage an over-used commons are increased aggregate profits and political viability. But, often implementation of such a system is postponed or never undertaken because the conditions are not ideal. One reason for this can be the absence of an inexpensive technology able to provide valid measurements of violations. However, there often is an inexpensive technology which is approximately valid. In this section, I study the possibilities for the design of a tradable permit system when the measurement technology involves an indirect measure of $q_{i}$ that contains statistical uncertainty. Such a measurement is imprecise.

I model imprecise measurement technologies as follows. The indirect measure of producer i's output is $w_{i}=q_{i}+\epsilon$ where $\epsilon$ is the measurement error. I assume $\epsilon$ is a random variable with density $f\left(\epsilon, q_{i}\right) d \epsilon$. Further, I assume that $\mathcal{E}\left(\epsilon \mid q_{i}\right)=\int \epsilon f\left(\epsilon, q_{i}\right) d \epsilon=0$; that is, the measurement technology is accurate. This is pretty much without loss of generality since, if $\mathcal{E}\left(\epsilon \mid q_{i}\right)$ is not accurate

\footnotetext{
${ }^{13}$ For details, see, e.g., Ledyard (2017).
} 
but is a known function of $q_{i}$, then one can adjust the penalty function to account for the inaccuracy. ${ }^{14}$ Finally, I assume that the measurement error is the same for every producer. This is not entirely without loss of generality. ${ }^{15}$

The measured violation is $w_{i}-l_{i}=\epsilon+q_{i}=v_{i}+\epsilon$. The penalty to be paid by the producer, based on the measured violation, is $\rho\left(w_{i}-l_{i}\right)$. When the producer chooses her output, she faces an expected penalty payment of $\mathcal{P}\left(v_{i}, q_{i}\right)=\int \rho\left(v_{i}+\epsilon\right) f\left(\epsilon, q_{i}\right) d \epsilon$.

With imprecise measurement, the definition of market equilibrium in Section 3 needs to be slightly altered to allow for the fact that the expected penalty now depends on $q_{i}$.

Definition 6 (Market equilibrium under Imprecise Measurement).

A permit market equilibrium under imprecise measurement is $\left(q^{*}, v^{*}, p^{*}, Q^{*}\right)$ such that,

(i) each producer $i$, chooses $\left(q_{i}^{*}, v_{i}^{*}\right)$ to solve

$$
\max _{\left(q_{i}, v_{i}\right)} b_{i}\left(q_{i}, Q^{*}\right)-p^{*}\left(q_{i}-v_{i}-l_{i}^{o}\right)-\mathcal{P}\left(v_{i}, q_{i}\right)
$$

(ii) $Q^{*}=\sum_{i} q_{i}^{*}$,

and

(iii) $\sum_{i} q_{i}^{*}-\sum_{i} v_{i}^{*}=L$.

$(q, Q)$ and $v$ are no longer independent as they were under valid measurement. But we can still define demand and supply functions.

Definition 7 (Supply and Demand under Imprecise Measurement).

Supply under imprecise measurement is $\left[q^{I}(p), Q^{I}(p)\right]$ and Demand under imprecise measurement is $\left[v^{I}(p), V^{I}(p)\right]$ where

\footnotetext{
${ }^{14}$ See Ledyard (2018).

${ }^{15}$ Differences across producers might occur in water markets for different crops or different irrigation technologies, and in fishing markets for different species or different gear types. These differences would affect the efficiency results below to some extent. I leave it to the reader to work out those implications.
} 


$$
\begin{aligned}
& q_{i}^{I}(p) \in \arg \max _{q_{i}} b_{i}\left(q_{i}, Q^{I}(p)\right)-p q_{i}-\mathcal{P}\left(v_{i}^{I}(p), q_{i}\right) \text { and } Q^{I}(p)=\sum_{i=1}^{N} q_{i}^{I}(p) . \\
& v_{i}^{I}(p) \in \arg \max _{v_{i}} p v^{i}-\mathcal{P}\left(v_{i}, q_{i}^{I}(p)\right) \text { and } V^{I}(p)=\sum_{i=1}^{N} v_{i}^{I}(p) .
\end{aligned}
$$

The market equilibrium price satisfies $Q^{I}\left(p^{*}\right)-V^{I}\left(p^{*}\right)=L$.

To ensure the market is well-behaved, I impose a regularity assumption.

Assumption 3 (Regular Imprecise Measurement).

\section{A. Errors}

(i) The indirect measure of output is $w_{i}=q_{i}+\delta h\left(q_{i}\right), \forall i$, where $\delta$ is a random variable with density $g(\delta)$ and $\mathcal{E}[\delta]=\int \delta g(\delta) d \delta=0$.

(ii) There is a $\underline{\delta}>0$ such that $\delta \geq-\underline{\delta}$ and $\frac{q}{h(q)} \geq \underline{\delta}$ for all $q \geq 0 .{ }^{16}$

(iii) The size of the errors, $h(q)$, is positive, non-decreasing, continuously differentiable, and convex in $q$. That is, $h\left(q_{i}\right)>0, h_{q}\left(q_{i}\right) \geq 0$ and $h_{q q}\left(q_{i}\right) \geq 0$, for all $q_{i}>0$. Also $h(0)=0$.

B. Enforcement

(i) $\rho\left(m_{i}\right)=\max \left\{0, a m_{i}\right\}$ with $a>0$, where $m_{i}=w_{i}-l_{i}$ is the measured violation.

(ii) $L>Q^{I}\left(P_{v}(0+)\right)=Q^{I}[a(1-G(0))]$.

Assumption 3A provides a structure that is like a single crossing property. It keeps the densities of the errors under control when $q$ changes. Increasing $h(q)$ is then like applying a mean-preserving spread to the error.

Assumption 3B is similar to Assumption 2 with a slight weakening. Assumption $3 \mathrm{~B}$ (ii) ensures that penalties are strong enough that producers will not want violations in equilibrium.

\footnotetext{
${ }^{16}$ This ensures that $w_{i} \geq 0$. If errors are proportional to output with $h(q)=\tau q$ for some $\tau>0$, then this will be true if $\tau \underline{\delta} \leq 1$.
} 
Under Assumption 3, the expected penalty function becomes

$$
\mathcal{P}\left(v_{i}, q_{i}\right)=a \int_{-\frac{v_{i}}{h\left(q_{i}\right)}}\left[v_{i}+\delta h\left(q_{i}\right)\right] g(\delta) d \delta .
$$

The geometry of a permit market under imprecise measurement satisfying Assumption 3 is illustrated in Figure 2 in Section 7.2. The crucial fact is that $Q^{I}(p)$ is downward sloping.

Result 3. Under Assumptions 1 and 3, $Q_{p}^{I}(p)<0$.

Proof. Let $k_{i}=-\frac{v_{i}}{h\left(q_{i}\right)}$, and $R\left(k_{i}\right)=\int_{k_{i}} \delta g(\delta) d \delta$. The first order conditions for a solution to Definition 6(i) and (ii) for a given $p$ are:

$$
\begin{aligned}
b_{i q}\left(q_{i}, Q\right)-p-h_{q}\left(q_{i}\right) a R(k) & =0 \\
p-a(1-G(k)) & =0 \\
v_{i}+h\left(q_{i}\right) k & =0 \\
\sum_{i} q_{i}-Q & =0 \\
\sum_{i} q_{i}-\sum_{i} v_{i} & =L
\end{aligned}
$$

To solve for the partial equilibrium comparative statics, differentiate (2)-(5) with respect to $p$ to get:

$$
\begin{aligned}
b_{i q q} q_{i p}^{I}+b_{i q Q} Q_{p}^{I}-1-h_{q q} a R q_{i p}^{I}-h_{q} a R_{k} k_{p}^{I} & =0 \\
1+a g k_{p}^{I} & =0 \\
v_{i p}^{I}+h_{q} k q_{i p}^{I}+h k_{p}^{I} & =0 \\
{\left[\sum_{i} q_{i p}^{I}\right]-Q_{p}^{I} } & =0 .
\end{aligned}
$$

From $(8), k_{p}^{I}(p)=-\frac{1}{a g(k)}<0$. By definition $R_{k}=-k^{I} g$. Since $v_{i}^{I}<0$, $k^{I}>0$. Solving (7) for $q_{i p}^{I}$ yields

$$
q_{i p}^{I}=\frac{1+h_{q} k-b_{i q Q} Q_{p}^{I}}{b_{i q q}-h_{q q} a R}=\alpha_{i}(p)-\beta_{i}(p) Q_{p}^{I}(p) .
$$


From (10)

$$
Q_{p}^{I}=\sum_{i} \alpha_{i}-\left(\sum_{i} \beta_{i}\right) Q_{p}
$$

Solving gives

$$
Q_{p}^{I}=\frac{\sum_{i} \alpha_{i}}{1+\sum_{i} \beta_{i}}
$$

Since $\alpha_{i}<0$ and $\beta_{i} \geq 0$, the result follows.

\subsection{Efficiency and Political Viability - Impossibility}

Although the equilibrium equations for valid measurement and imprecise measurement look very similar, the latter create serious problems for both the efficiency and the political viability of permit programs.

\subsubsection{Efficiency}

Efficiency given $L$ requires that, at a permit market equilibrium $\left(q^{*}, v^{*}, p^{*}, Q^{*}\right)$, $v_{i}^{*}=0$ for all producers. With imprecise measurement, under Assumptions 1 and 3 , this will not be true. Instead, $v_{i}^{*}<0$ for all $i$ and, therefore, $Q^{*}<L$ in equilibrium.

Result 4. Under Assumptions 1 and 3, at a permit market equilibrium $\left(q^{*}, v^{*}, p^{*}, Q^{*}\right), 0<p^{*}<a[1-G(0)], v_{i}^{*}<0, \forall i$, and $Q^{*}<L$.

Proof. $\left(p^{*}<a[1-G(0)]\right)$ Assume the contrary. Then $v_{i}^{*}>0$ and, so, $Q^{*}>L$. But $Q\left(p^{*}\right) \leq Q(a[1-G(0)])<L$ which is a contradiction.

$\left(v_{i}^{*}<0\right)$ From profit maximization $v_{i}^{*} \in \arg \max _{v_{i}} p^{*} v_{i}-\int_{\frac{-v_{i}}{h\left(q_{i}\right)}} a\left(v_{i}+\delta h\left(q_{i}\right)\right) g(\delta) d \delta$.

The first order condition for this is $p^{*}=a \int_{-\frac{v_{i}}{h\left(q_{i}\right)}} g(\delta) d \delta=a\left[1-G\left(-\frac{v_{i}}{h\left(q_{i}\right)}\right)\right]$. Since $p^{*}<a[1-G(0)]$, it follows that $v_{i}^{*}<0$. This implies $Q^{*}<L$.

With imprecise measurement, producers will pay a penalty even if $q=l$. They can reduce that expected penalty cost by producing less than the permits they hold. Because of this, if regulators issue a total of permits $L$ equal 
to the desired aggregate output target, $Q$, actual output will be less than $Q$ and, therefore, not efficient given $Q$.

Knowing that equilibrium output is less than $L$, one might ask whether regulators could change $L$ to some other $\hat{L}$ so as to move $Q^{I}\left(p^{*}\right)$ to $L$. For the regular case, the answer is yes. Consider Figure 2. The amount of permits that works is $\hat{L}=Q^{I}\left(p^{V}\right)-V^{I}\left(p^{V}\right)>L$. There must be enough permits to allow the producer to use the extras to insure herself against a faulty measurement. That is, an additional amount $\hat{L}-L^{*}=-V^{I}\left(p^{V}\right)$ must be

added to $L^{*}$. Of course, to compute $\hat{L}$ the regulator must know, prior to the implementation of the program, the functions $Q^{I}(p)$ and $V^{I}(p)$ which they do not.

\subsubsection{Political Viability}

With valid measurement, by Result $2 \mathrm{~B}$, there are distributions of the initial permit, $l^{\circ}$, such that all producers will be at least as well off in the permit market equilibrium as they would have been without the program. With imprecise measurement, this may not be true because a producer's expected penalties are positive even if she chooses to buy licenses equal in number to her production levels. That is, $\mathcal{P}\left(0, q_{i}\right)=a h\left(q_{i}\right) \int_{0} \delta g(\delta) d \delta>0$.

To see the effect of imprecise measurement on political viability, consider the following result which adapts Result 2B to imprecise measurement.

Result 5. Under Assumptions 1 and 3,

(i) for any initial allocation of permits, $l^{o}$, such that $b_{i}\left(l_{i}^{o}, L\right)-\mathcal{P}\left(0, l_{i}^{o}\right)>$ $b_{i}\left(q_{i}^{a}, Q^{a}\right)$, at the market equilibrium $\left(q^{*}, v^{*}, p^{*}, Q^{*}\right)$

$$
b_{i}\left(q_{i}^{*}, Q^{*}\right)-p^{*}\left(q_{i}^{*}-v_{i}^{*}-l_{i}^{o}\right)-\mathcal{P}\left(v_{i}^{*}, q_{i}^{*}\right)>b_{i}\left(q_{i}^{a}, Q^{a}\right), \forall i,
$$

and 
(ii) there exists an $l^{\circ}$ satisfying (i) if and only if there is a $\hat{q}$ such that $\sum_{i} \hat{q}=$ $L$ and $\left.\sum_{i} b_{i}(\hat{q}, L)-\sum_{i} b_{i}\left(q^{a}, Q^{a}\right)>\sum_{i} \mathcal{P}\left(0, \hat{q}_{i}\right)\right]$.

Proof. (i) Let $\left(q^{*}, v^{*}, p^{*}, Q^{*}\right)$ be the permit market equilibrium. Then $b_{i}\left(q_{i}^{*}, Q^{*}\right)-$ $p^{*}\left(q_{i}^{*}-v_{i}^{*}-l_{i}^{o}\right)-\mathcal{P}\left(v_{i}^{*}, q_{i}^{*}\right) \geq b_{i}\left(l_{i}^{o}, Q^{*}\right)-p^{*}\left(l_{i}^{o}-0-l_{i}^{o}\right)-\mathcal{P}\left(0, l_{i}^{o}\right) \geq b_{i}\left(l_{i}^{o}, L\right)-$ $\mathcal{P}\left(0, l_{i}^{o}\right)>b_{i}\left(q_{i}^{a}, Q^{a}\right)$. The first inequality follows from profit maximization. The second follows because $L>Q(a[1-G(0)])$ implies $Q^{*} \leq L$. The last follows from the assumption on $l^{\circ}$.

Comparing this to Result 2 under valid measurement, it is easy to see that imprecise measurement introduces a potential barrier to voluntary participation. If either the penalty rate or the errors are large, the expected penalty with no violations, $a h\left(q_{i}\right) \int_{0} \delta g(\delta) d \delta$, is large. Then, if the gains from improving the management of the commons are not very large, it may be difficult or impossible to find an appropriate $l_{i}^{o}$.

\subsection{Efficiency and Political Viability - Possibility}

In spite of the difficulties described in the previous section, some positive results can be found.

\subsubsection{Efficiency}

Although there is no general efficiency result when measurement is imprecise, if measurement errors are proportional to output, then even though $q^{*}$ is not efficient given $L, q^{*}$ is efficient given $Q^{*}$. That is, production will be organized efficiently given the aggregate output level.

Assumption 4. (Errors are proportional to output)

$$
h_{q q}\left(q_{i}\right)=0, \forall q, \forall i .
$$

This assumption along with Assumption $3 \mathrm{~A}(\mathrm{iii})$ imply that $h(q)=\tau q$ for some $\tau>0$. 
Result 6. Under Assumptions 1, 3 and $4, q^{*}$ is efficient given $Q^{*}$.

Proof. Under Assumptions 1 and 3, the FOC for a permit market equilibrium are found in (2)-(6). From (3) it follows that $k^{*}$ is the same for all $\mathrm{i}$. Therefore, from (2) and (4), $q^{*}$ is efficient given $Q^{*}$ if and only if the $h_{q}\left(q_{i}^{*}\right)$ are equal for each i. This is true under Assumption 4 since $h_{q q}\left(q_{i}\right)=0$.

Without Assumption 4, the equilibrium will not be efficient given $Q^{*}$. It is easy to see why. If $h_{q q}>0$, then $h_{q}(\hat{q})>h_{q}(\tilde{q})$ iff $\hat{q}>\tilde{q}$. Let $\bar{h}_{q}=\frac{\sum h_{q}\left(q_{i}^{*}\right)}{N}$ be the average value of $h_{q}$ in equilbrium. If $h_{q}\left(q_{i}^{*}\right)>\bar{h}_{q}$ then in equilibrium $q_{i}^{*}$ is relatively smaller than desired for efficiency. If $h_{q}\left(q_{i}^{*}\right)<\bar{h}_{q}$ then in equilibrium $q_{i}^{*}$ is relatively larger than desired for efficiency. The fact that the imprecise measurement errors are getting worse as q gets larger means that those who produce a large amount will have more incentive to cut back on their output to avoid the penalties from mis-measurement. Non-proportional measurement errors interfere with efficiency given $Q^{*}$.

\subsubsection{Political Viability}

There is a way to design around this problem by using the same insights employed for valid measurement. Put the producer in an initial position that is at least as good as autarky and that she can protect. If the regulator gives each producer an initial subsidy of $P_{i}^{o}=\mathcal{P}\left(0, l_{i}^{o}\right)$, and if the producer then chooses $\left(q_{i}, v_{i}\right)=\left(l_{i}^{o}, 0\right)$, she can guarantee that her expected penalty less the subsidy will be zero. That plus the appropriate initial permit allocation guarantees voluntary participation.

Result 7. Under Assumptions 1 and 3, for any initial allocation of permit, $l^{o}$, such that $b_{i}\left(l_{i}^{o}, L\right)>b_{i}\left(q_{i}^{a}, Q^{a}\right)$ for all $i$, there are lump-sum payments $P_{i}^{o}=\mathcal{P}\left(0, l_{i}^{o}\right)$ such that the market equilibrium $\left(q^{*}, v^{*}, p^{*}, Q^{*}\right)$ satifies

$$
b_{i}\left(q_{i}^{*}, Q^{*}\right)-p^{*}\left(q_{i}^{*}-v_{i}^{*}-l_{i}^{o}\right)-\mathcal{P}\left(v_{i}^{*}, q_{i}^{*}\right)+P_{i}^{o}>b_{i}\left(q_{i}^{a}, Q^{a}\right), \forall i .
$$

Proof. Let $\left(q^{*}, v^{*}, p^{*}, Q^{*}\right)$ be the permit market equilibrium. Then $b_{i}\left(q_{i}^{*}, Q^{*}\right)-$ $p^{*}\left(q_{i}^{*}-v_{i}^{*}-l_{i}^{o}\right)-\mathcal{P}\left(v_{i}^{*}, q_{i}^{*}\right)+P_{i}^{o} \geq b_{i}\left(l_{i}^{o}, Q^{*}\right)-p^{*}\left(l_{i}^{o}-0-l_{i}^{o}\right)-\mathcal{P}\left(0, l_{i}^{o}\right)+P_{i}^{o} \geq$ $b_{i}\left(l_{i}^{o}, L\right)>b_{i}\left(q_{i}^{a}, Q^{a}\right)$. The first inequality follows from profit maximization. 
The second follows because $L>Q(a[1-G(0)])$ implies $Q^{*} \leq L$. The last follows from the assumption on $l^{o}$ and the fact that $\mathcal{P}\left(0, l_{i}^{o}\right)=P_{i}^{o}$.

If the measurement technology is well understood, then $P_{i}^{o}=a h\left(l_{i}^{o}\right) \int_{0} \delta g(\delta) d \delta$ is easy to calculate.

Although the subsidies in Result 7 help solve the problem of generating voluntary participation, they create a new problem for the designers. If the subsidies are deployed and equilibrium output is less than or equal to $L$, then producers will be receiving a positive net aggregate subsidy. The permit program will not be self financing in expected value.

Result 8. Under Assumptions 1, 3, and Assumption 4, $\sum_{i} \mathcal{P}\left(0, l_{i}^{o}\right)>$ $\sum_{i} \mathcal{P}\left(v_{i}^{*}, q_{i}^{*}\right)$.

Proof. Under Assumptions 3 and 4, in equilibrium $\sum_{i}\left[\mathcal{P}\left(0, l_{i}^{o}\right)-\mathcal{P}\left(v_{i}^{*}, q_{i}^{*}\right)\right]=$ $a \tau l_{i}^{o} \int_{0} \delta g(\delta) d \delta-a \tau q_{i}^{*} \int_{\frac{-v^{*}}{h\left(q_{i}^{*}\right)}} \delta g(\delta) d \delta$. By $(3), \int_{\frac{-v^{*}}{h\left(q_{i}^{*}\right)}} \delta g(\delta) d \delta=\frac{p^{*}}{a}$ is the same for every i. Therefore the aggregate net subsidy is $S=\sum_{i}\left[\mathcal{P}\left(0, l_{i}^{o}\right)-\mathcal{P}\left(v_{i}^{*}, q_{i}^{*}\right)\right]=$ $a \tau\left[\sum_{i} l_{i}^{o}\right] \int_{0} \delta g(\delta) d \delta-a \tau\left[\sum_{i} q_{i}^{*}\right] \int_{\frac{-v^{*}}{h\left(q_{i}^{*}\right)}} \delta g(\delta) d \delta=a \tau\left[L \int_{0} \delta g(\delta) d \delta-Q^{*} \int_{\frac{-v^{*}}{h\left(q_{i}^{*}\right)}} \delta g(\delta) d \delta\right]$.

Under Assumption 3, $v_{i}^{*}<0$ which implies that $\int_{0} \delta g(\delta) d \delta>\int_{\frac{-v^{*}}{h\left(q_{i}^{*}\right)}} \delta g(\delta) d \delta$. Also $L>Q^{*}$. Therefore, $S>0$.

The voluntary participation of the producers has been bought with funding from outside of the market - presumably from taxpayers. This creates a new friction against adoption. Nevertheless, such subsidies may be justified. The rationalization of the management of the commons can lead to gains, not only for producers, but also for the consumers of the products of the commons. This is certainly true for fisheries, and probably true for many other situations. Using some of these gains to ease the transition to permit markets might be a very good bargain for all concerned.

\subsection{Precision}

In this section, I explore what happens to efficiency and political viability as the measurements become more precise. The easiest way to do that is 
to introduce a precision parameter, $\eta$ and replace $h\left(q_{i}\right)$ with $\eta h\left(q_{i}\right)$ in the measurement model. Thus, $w_{i}=q_{i}+\eta h\left(q_{i}\right) \delta$. I will assume $\eta \leq 1$. With this small change, the measurement error is $w_{i}-q_{i}=z h\left(q_{i}\right)$ where $z=\delta \eta$. The expected value of $\mathrm{z}$ is 0 and the variance of $\mathrm{z}$ is $\eta \operatorname{Var}(\delta)$. As $\eta$ decreases, precision increases.

The penalty that a producer now faces, given output $q_{i}$ and actual violation $v_{i}=q_{i}-l_{i}$, is

$$
\mathcal{P}^{\eta}\left(v_{i}, q_{i}\right)=a \int_{-\frac{v_{i}}{\eta h\left(q_{i}\right)}}\left[v_{i}+\delta h\left(q_{i}\right) \eta\right] g(\delta) d \delta
$$

As one might expect, increased precision improves efficiency and eases political viability. In the limit as $\eta \rightarrow 0$, the equilibrium under imprecise measurement approaches the equilibrium under valid measurement. The permit market equilibrium becomes efficient given L and no subsidies are required for voluntary participation.

Result 9. Let $(q(\eta), v(\eta), p(\eta), Q(\eta))$ be the market equilibrium under imprecise measurement with the expected penalty $\mathcal{P}^{\eta}\left(v_{i}, q_{i}\right)=a \int_{-\frac{v_{i}}{\eta h\left(q_{i}\right)}}\left[v_{i}+\right.$ $\left.\delta h\left(q_{i}\right) \eta\right] g(\delta) d \delta$ and let $\left(q^{*}, v^{*}, p^{*}, Q^{*}\right)$ be the market equilibrium under valid measurement with the penalty $P\left(v_{i}\right)=\max \{a v, 0\}$. Under Assumptions 1 and 3,

(i) $\lim _{\eta \rightarrow 0}(q(\eta), v(\eta), p(\eta), Q(\eta))=\left(q^{*}, v^{*}, p^{*}, Q^{*}\right)$ and

(ii) $\lim _{\eta \rightarrow 0} \mathcal{P}^{\eta}\left(0, l_{i}^{o}\right)=0$.

Proof. First note that, as precision increases, the penalty function under imprecise measurement approaches the penalty function under valid measurement. For any $q_{i}$ such that $0<q_{i}<\infty$,

$$
\begin{gathered}
\lim _{\eta \rightarrow 0} \mathcal{P}^{\eta}\left(v_{i}, q_{i}\right)=a v \text { if } v \geq 0 \\
\lim _{\eta \rightarrow 0} \mathcal{P}^{\eta}\left(v_{i}, q_{i}\right)=0 \text { if } v \leq 0 .
\end{gathered}
$$

The first order conditions for a market equilibrium under imprecise measure- 
ment are:

$$
\begin{array}{r}
b_{i q}\left(q_{i}, Q\right)-p-\mathcal{P}_{q_{i}}^{\eta}\left(v_{i}, q_{i}\right)=0 \\
p-\mathcal{P}_{v_{i}}^{\eta}\left(v_{i}, q_{i}\right)=0 \\
\sum_{i} q_{i}-Q=0 \\
\sum_{i} q_{i}-\sum_{i} v_{i}=L
\end{array}
$$

It is easy to show that $\mathcal{P}_{q_{i}}^{\eta}\left(v_{i}, q_{i}\right) \rightarrow 0$ as $\eta \rightarrow 0$. Also $\mathcal{P}_{v_{i}}^{\eta}\left(v_{i}, q_{i}\right) \rightarrow a$ as $\eta \rightarrow 0$ if $v>0$ and $\mathcal{P}_{v_{i}}^{\eta}\left(v_{i}, q_{i}\right) \rightarrow 0$ as $\eta \rightarrow 0$ if $v^{i}<0$. Result 9(i) follows from the implicit function theorem.

$\mathcal{P}^{\eta}\left(0, l_{i}^{o}\right)=\int_{0} \delta h\left(l_{i}^{o}\right) \eta g(\delta) d \delta=\eta h\left(l_{i}^{o}\right) \int_{0} \delta g(\delta) d \delta=\eta \mathcal{P}\left(0, l_{i}^{o}\right)$. Result 9(ii) follows.

\section{Final Comments}

In this paper I have explored the geometry, efficiency and political viability of permit market equilibria when enforcement can only use imprecise measurement. I provide a set of conditions (Assumption 3) such that the geometry corresponds to most economists' intuitions. These conditions are similar in spirit to those needed under accurate measurement (Assumption 2).

Unlike the standard results when there is accurate and precise measurement of use, when measurement is imprecise permits will not be efficiently allocated. There are two sources of the inefficiency: aggregate output is less than the number of permits as producers overbuy to insure against mismeasurement and there can be bias in the efficient allocation of output as mis-measurement can have different marginal effects on producers depending on their size. Further, when measurement is imprecise, it may not be possible to find an initial allocation of permits such that all firms are better off than they were before implementation of the program. The reason is that all firms will face positive expected penalty payments, even if they have negative actual violations. This could seriously affect the political viability of the program, even if measurement were free. 
But there are also some positive results. First, if the errors are proportional to output, then aggregate output will be efficiently allocated among firms. The only inefficiency will be that aggregate output is less than the target, the number of permits. This can be compensated for by issuing more permits than the target output. Second, there are easily calculated individual subsidies that will make it possible to find an initial allocation of permits to guarantee voluntary participation. This will mean that taxpayers must subsidize the implementation of the program. But if the benefits from the program are large enough and some of these benefits accrue to consumers, then the program may still be politically viable.

Finally, if the measurement errors are small, inefficiencies will be small and the subsidies required for voluntary participation will be small. In this case, it may well be reasonable to proceed with an inexpensive, imprecise measurement system as if it were accurate. 


\section{$6 \quad$ References}

Cason, T. N. and L. Gangadharan (2006) "Emissions variability in tradable permit markets with imperfect enforcement and banking", Journal of Economic Behavior and Organization, 61(2), 199-216.

Hurwicz, L. (1960) "Optimality and Informational Efficiency in Resource Allocation Processes", Proceedings of a Symposium on Mathematical Methods in the Social Sciences, 1959, Stanford University Press, 27-46.

Hurwicz, L. (1972) "On informationally decentralized systems", in R. Radner and C. B. McGuire, eds. Decisions and Organizations a volume in honor of Jacob Marschak, North Holland, 297-336.

Hurwicz, L. and S. Reiter (2006) Designing Economic Mechanisms, Cambridge University Press.

Keeler, A. (1991) "Noncompliant firms in transferable discharge permit markets: Some extensions", Journal of Environmental Economics and Management, 21(2), 180-189.

Ledyard, J. (2018) "Imprecise Measurement and Tradable Permits: Geometry and Comparative Statics", Working Paper, Caltech.

Malik, A. S. (1990) "Markets for pollution control when firms are noncompliant", Journal of Environmental Economics and Management, 18(2), 97-106.

Malik, A. S. (1992) "Enforcement costs and the choice of policy instruments for controlling pollution", Economic Inquiry, 30(4), 714-721.

Murphy, J. J. and J. K. Stranlund (2007) "A laboratory investigation of compliance behavior under tradable emissions rights: Implications for targeted enforcement", Journal of Environmental Economics and Management, 53(2), 196-212.

Stranlund, J. K. (2007) "The regulatory choice of noncompliance in emissions trading programs", Environmental and Resource Economics, 38(1), 99-117.

Stranlund, J. K. and K. K. Dhanda (1999) "Endogenous monitoring and enforcement of a transferable emissions permit system", Journal of Environmental Economics and Management, 38(3), 267-282. 
Stranlund, J. K. J. J. Murphy and J. M. Spraggon (2008) "Imperfect Enforcement of Emissions Trading and Industry Welfare: A Laboratory Investigation", working paper, March

Water Education Foundation, (2015) "The View from Above: The Promise of Remote Sensing", http://www.watereducation.org/western-water-excerpt/viewabove-promise-remote-sensing.

Zekri, S. (2009) "Controlling groundwater pumping online", Journal of Environmental Management. 90(11), 3581-8. 


\section{$7 \quad$ Figures and Comments}

\subsection{Valid Measurement}

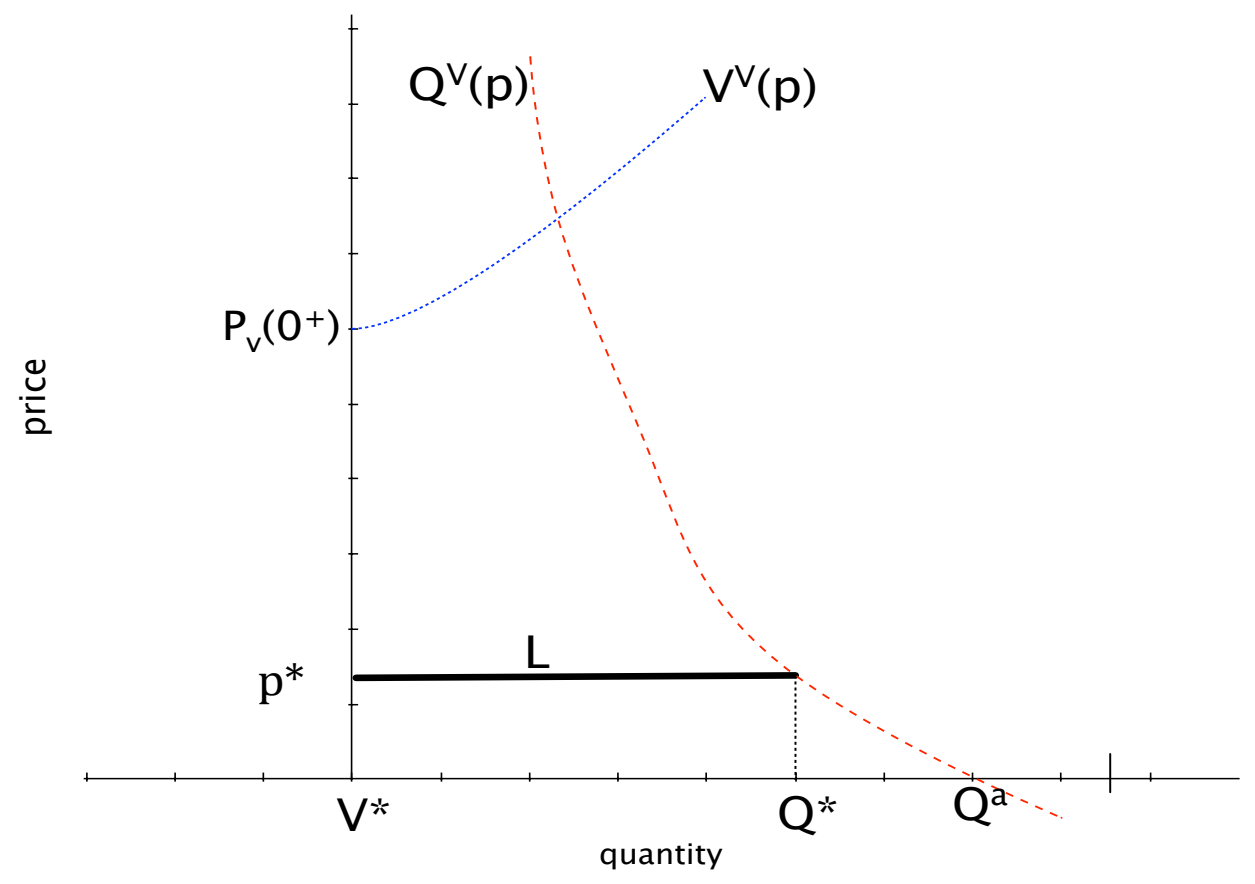

Figure 1: Market Equilibrium with Valid Measurement

Figure 1 displays the standard situation for a permit market equilibrium under valid measurement. The equilibrium under autarky is $Q^{a}$. $P_{v}\left(0^{+}\right)=$ $\lim _{v \rightarrow 0^{+}} d P(v) / d v$, the right-hand derivative of the penalty function, is the intercept of $V^{V}(p)$.

The dashed red line, denoted $Q^{V}(p)$, is the partial equilibrium aggregate supply of output of the commons. The dashed blue line, denoted $V^{V}(p)=$ $N P_{v}^{-1}(p)$, is the partial equilibrium aggregate demand for violations.

The equilibrium price of the permit market is $p^{*}$. Since $L \geq Q^{V}\left(P_{v}\left(0^{+}\right)\right)$, equilibrium demand is $V^{*}=V^{V}\left(p^{*}\right)=0$ and equilibrium supply is $Q^{*}=$ $Q^{V}\left(p^{*}\right)=L$. 


\subsection{Imprecise Measurement}

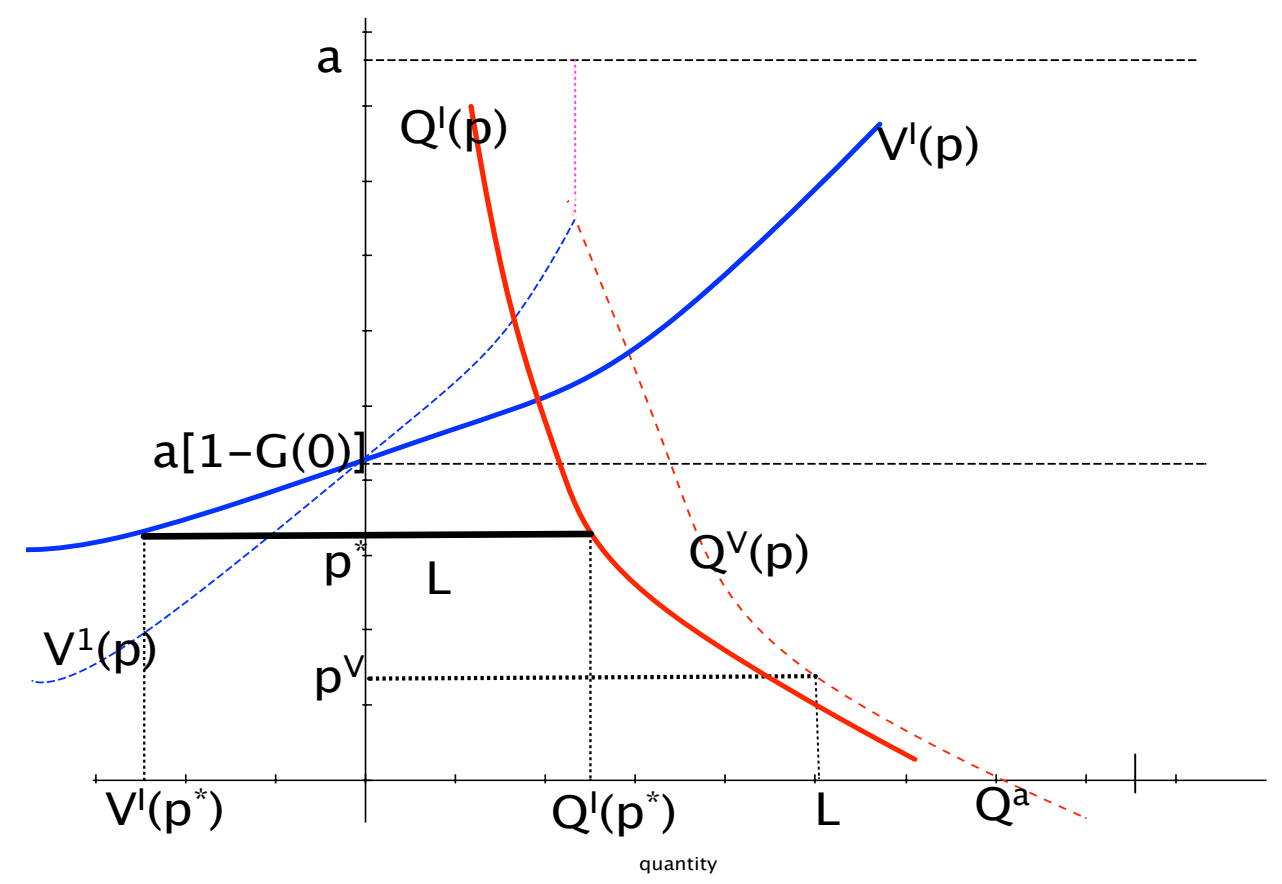

Figure 2: Market Equilibrium with Imprecise Measurement

The dashed red line, $Q^{V}(p)$, comes from Figure 1 and is the aggregate supply under valid measurement. $p^{V}$ is the equilibrium price under valid measurement. The dashed blue line, $V^{1}(p)$, is the partial equilibrium aggregate demand for violations if measurement errors are independent of $q_{i}$. That is $V^{1}(p)=\sum_{i} v_{i}^{1}(p)$ where $v_{i}^{1}(p)=\arg \max _{v_{i}} p v_{i}-a \int_{-v_{i}}\left(v_{i}+\delta\right) g(\delta) d \delta$.

The solid blue and red lines, $Q^{I}(p)$ and $V^{I}(p)$, are the partial equilibrium supply and demand from Definition 7 . Because a penalty is assessed for measured violations larger than actual violations, $Q^{I}(p)$ lies to the left of aggregate demand under accurate measurement. Because $V^{I}(p)=-\left[\sum_{i} h\left(q_{i}\right)\right] G^{-1}\left(1-\frac{p}{a}\right)$, $V^{I}(p)$ rotates clockwise from $V^{1}(p){ }^{17}$

\footnotetext{
${ }^{17}$ This is drawn for the case that $\frac{\left[\sum_{i} h\left(q_{i}\right)\right]}{N}>1$. If that is not true, then $V(p)$ rotates in a counter-clockwise direction.
} 
$p^{*}$ is the equilibrium price under imprecise measurement. The equilibrium aggregate supply of output is $Q^{*}=Q^{I}\left(p^{*}\right)<L$. The equilibrium aggregate demand for violations is $V^{*}=V^{I}\left(p^{*}\right)<0$ because excess permits are held in equilibrium. $V^{*}+L^{*}=Q^{*}$. 